\title{
Wenchuan earthquake ruptures located by offset-tracking procedure of ENVISAT ASAR amplitude images*
}

\author{
Bin Liu $^{1,2, \uparrow}$ Wenliang Jiang ${ }^{2}$ Jingfa Zhang ${ }^{2}$ Yi Luo $^{2}$ and Lixia Gong ${ }^{2}$ \\ ${ }^{1}$ Institute of Engineering Mechanics, China Earthquake Administration, Harbin 150080, China \\ ${ }^{2}$ Institute of Crustal Dynamics, China Earthquake Administration, Beijing 100085, China
}

\begin{abstract}
Two rupture zones of Wenchuan earthquake have been successfully located by an offset-tracking procedure of ENVISAT ASAR amplitude images. Accuracy of offset-tracking strongly depends on the quality of image coregistration. In order to remove noise caused by coregistration, a combined filter method is proposed to remove noise of estimated offsets. In addition, Kriging interpolation is applied to smooth the azimuth displacement offset map. The displacement offset maps of azimuth and range show that about $245 \mathrm{~km}$ and $60 \mathrm{~km}$ in length along Beichuan-Yingxiu fault and Guanxian-Jiangyou fault were ruptured by Wenchuan earthquake, respectively. The result of offset-tracking is validated by field observations of China Earthquake Administration.
\end{abstract}

Key words: Wenchuan earthquake; offset-tracking; ENVISAT ASAR; InSAR

CLC number: P315.5 Document code: A

\section{Introduction}

The $M_{\mathrm{S}} 8.0$ Wenchuan earthquake caused great damages in Sichuan, Gansu and Shaanxi provinces of China. The earthquake broke about $240 \mathrm{~km}$ of Beichuan-Yingxiu fault, $72 \mathrm{~km}$ of Guanxian-Jiangyou fault and $6 \mathrm{~km}$ of Xiaoyudong fault, with a complex thrust-dextral slip mechanism (Xu et al, 2008). Figure 1 gives the distribution of faults in Wenchuan area, which were originally made by Deng et al (2007) and then complemented using ETM/TM and SAR intensity images in this paper.

Interferometric Synthetic Aperture Radar (InSAR) has an advantage of detecting ground displacement with high accuracy. However, as for the Wenchuan earthquake, because coherence between interferometric images are lost seriously due to large displacement amplitude along ruptures, InSAR is not helpful to understanding the faults directly related to the seismic rupture (Ge et al, 2008; Shan et al, 2008; Sun et al, 2008). Thus,

\footnotetext{
* Received 15 March 2010; accepted in revised form 28 April 2010; published 10 June 2010.

× Corresponding author. e-mail: lbin0226@163.com

(C) The Seismological Society of China and Springer-Verlag Berlin Heidelberg 2010
}

in order to reveal the unknown surface displacements of the Wenchuan area, some scholars conducted an offset-tracking procedure that can detect large ground displacements even in an incoherent area. de Michele et al (2010) located the Wenchuan earthquake ruptures using PALSAR L-band spaceborne synthetic aperture radar

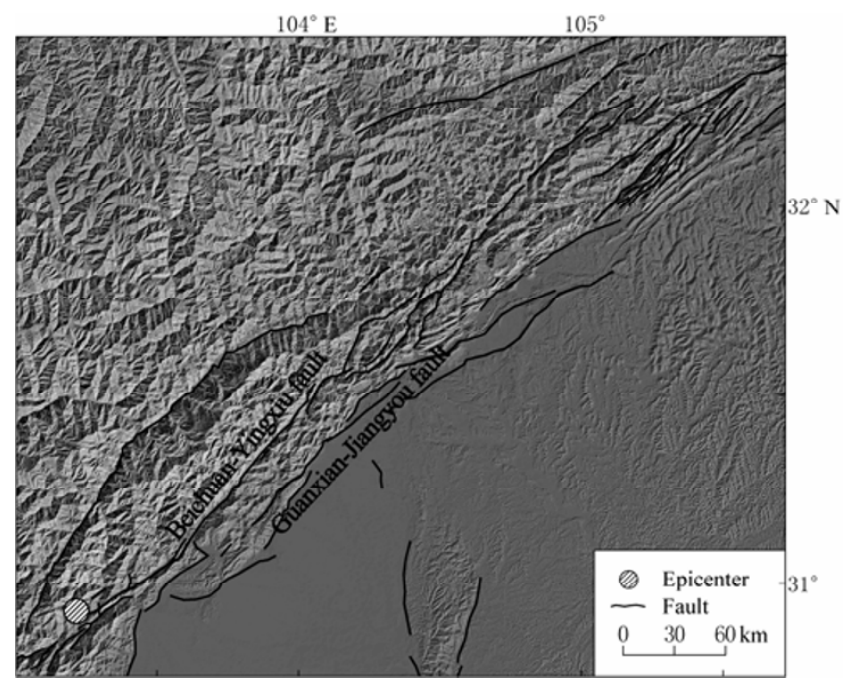

Figure 1 Fault distribution of Wenchuan area. The faults were originally made by Deng et al (2007), and then complemented using ETM/TM and SAR intensity images in this paper. 
(SAR) amplitude images. A sub-pixel correlation method was used to retrieve the coseismic displacement field projected into the line of sight of the satellite and the horizontal along track direction, and then to map the surface ruptures. Kobayashi et al (2009) detected detailed ground displacements in the proximity of the Longmenshan fault zone by applying a PALSAR offset-tracking method. An elevation-dependent correction is indispensable for achieving sub-meter accuracy.

Unfortunately, ionospheric anomalies are more clearly visible in the original azimuth offset field of PALSAR, and the swath width of PALSAR strip-map image is less than $70 \mathrm{~km}$. Both of them greatly increase the estimation errors. In this paper, we locate Wenchuan earthquake ruptures using C-band spaceborne synthetic aperture radar (SAR) from ASAR sensor onboard the ENVISAT satellite, and analyze the geometrical and kinematical characteristics of the Beichuan-Yingxiu fault and Guanxian-Jiangyou fault.

\section{Data and method}

Offset-tracking procedure is a method to estimate the range and azimuth displacement by sub-pixel registration of two SAR images. We use ENVISAT ASAR data on the descending orbital paths 290, 18 and 247 (Table 1). The obtained data is strip-map image with an incidence angle of $22.9^{\circ}$. We process the SAR data from original signal or SLC product using the routine implemented in the GAMMA software (Strozzi et al, 2002). The follow-up processing is implemented in Matlab.

Table 1 List of ENVISAT ASAR data acquired in Wenchuan earthquake

\begin{tabular}{cccc}
\hline \multirow{2}{*}{ Track } & $\begin{array}{c}\text { Data } \\
\text { a-mo-d }\end{array}$ & $\begin{array}{c}\text { Pixel size of range } \\
/ \mathrm{m}\end{array}$ & $\begin{array}{c}\text { Pixel size of azimuth } \\
/ \mathrm{m}\end{array}$ \\
\hline \multirow{2}{*}{290} & $2008-03-03$ & 7.8 & 4 \\
& $2008-06-16$ & 7.8 & 4 \\
\hline \multirow{2}{*}{018} & $2006-11-15$ & 7.8 & 4 \\
& $2008-05-28$ & 7.8 & 4 \\
\hline \multirow{2}{*}{247} & $2007-11-16$ & 7.8 & 4 \\
& $2008-08-22$ & 7.8 & 4 \\
\hline
\end{tabular}

In this paper the offset fields of azimuth and range are generated with a normalized cross-correlation of image patches of detected real-valued SAR intensity images. The location of the peak of two-dimensional cross-correlation function yields the image offset. After conducting coregistration between two amplitude images acquired before and after the earthquake, we divide the single-look SAR amplitude images into patches and calculate an offset between the corresponding patches by a normalized cross-correlation procedure (Kobayashi et al, 2009).

Compared with flat terrain, it is easier to yield noise of estimated offsets in an area with complex topography and large displacement. In order to produce a displacement offset map, many thousands of locations are measured. To distinguish good data from bad ones, a large correlation window (512 pixels $\times 512$ pixels) is used to reduce the occurrence of bad offset data. Unfortunately, this needs much longer computation times and great memory requirements. We estimate the sub-pixel offset of the full resolution images with step of 55 pixels in range and 472 pixels in azimuth direction, yielding approximately 50000 offsets vectors. Further, to reduce noise of the offset estimates, we take the ratio of the peak of the correlation function to their average value as signal-to-noise ratio (SNR) (Strozzi et al, 2002; Werner et al, 2005), and offset estimates with an SNR below the designated threshold are rejected for the final fit. Finally, polynomial models of range and azimuth offsets are obtained with linear least-squares estimation, i.e., equation (1). Once coefficient matrix is determined, a second criterion for rejection of values is based upon the deviation between offset estimates and the fitted values, and offset estimates with higher deviation will be rejected.

$$
\left\{\begin{array}{l}
r_{\text {offset }}=a_{1} r_{M}^{2}+b_{1} r_{M} a_{M}+c_{1} a_{M}^{2}+d_{1} r_{M}+e_{1} a_{M}+f_{1} \\
a_{\text {offset }}=a_{2} r_{M}^{2}+b_{2} r_{M} a_{M}+c_{2} a_{M}^{2}+d_{2} r_{M}+e_{2} a_{M}+f_{2}
\end{array},\right.
$$

where $r_{M}$ and $a_{M}$ are the range and azimuth coordinates of the master image, respectively; $r_{\text {offset}}, a_{\text {offset }}$ are the offsets of range and azimuth.

The image offsets in the range and azimuth directions are related to satellite orbit configurations of two SAR images, displacement between acquisition time intervals of the image pair, and ionospheric effects. The orbital offsets are determined by fitting a bilinear polynomial function [equation (2)] to offset fields computed globally from the SAR images, assuming no displacement for most parts of the image (Strozzi et al, 2006). Once systematic errors have been removed, coarse displacement offsets are generated. Then a simple directional filter is very efficient in getting rid of the remaining misregistration points, which was originally implemented by Kääb (2005). In this paper an improved adaptive directional filter is proposed to further remove the remaining noise. A displacement vector map calculated by coarse displacement offsets of azimuth and range is firstly produced before filtering, and then a 
small filter window ( 5 pixels $\times 5$ pixels) will be used in the coarse displacement vector map. In the 5 pixels $\times 5$ pixels window, the points with displacement direction changing abruptly will be excluded. Additionally, ionospheric anomalies are clearly visible in the azimuth offset field but hardly visible in the range offset field (Wegmüller et al, 2006). Azimuth displacement offsets of all points are discrete in the map due to ionospheric anomalies (Figure 3a, right), so we smooth the azimuth displacement offset map by Kriging interpolation. Figure 2 gives the processing flow of displacement offset map generating.

$$
\left\{\begin{array}{l}
r_{\text {offset }}=a_{1} r_{M} a_{M}+b_{1} r_{M}+c_{1} a_{M}+d_{1} \\
a_{\text {offset }}=a_{2} r_{M} a_{M}+b_{2} r_{M}+c_{2} a_{M}+d_{2},
\end{array}\right.
$$

where $r_{M}, a_{M}$ are the range and azimuth coordinates respectively of the master image; $r_{\text {offset }}, a_{\text {offset }}$ are the offsets of range and azimuth in areas without displacement.

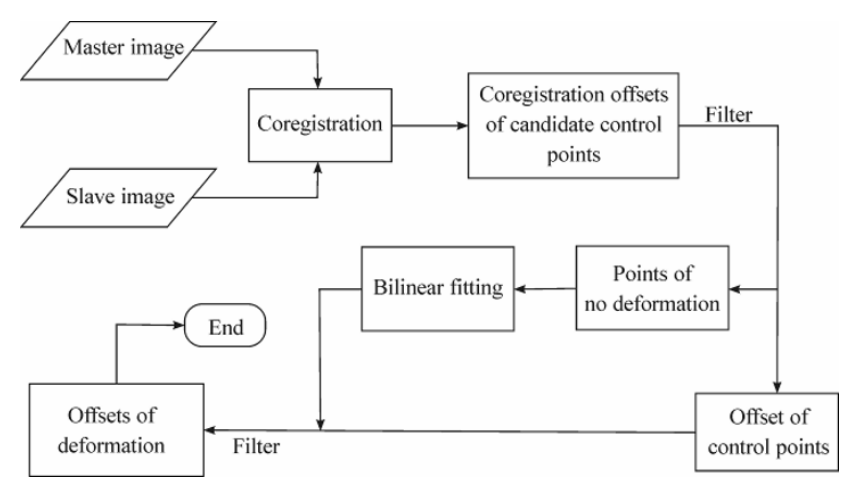

Figure 2 Processing flow of displacement offset map generating.

\section{Results and analyses}

The offset fields generated from coregistering real-valued SAR intensity images show an obvious bilinear relation (Figure 3a). The points around faults and those with low SNR are excluded in order to fit a bilinear polynomial function (Figure 3b). Residual offsets in range and azimuth correspond to ground displacement along the line of sight and the satellite track, respectively. This yields relative accuracy of $0.4 \mathrm{~m}$ in azimuth and $0.2 \mathrm{~m}$ in range.

The range and azimuth displacement offset fields over the Wenchuan area are shown in Figure 4. In the range displacement offset map (Figure 4a), blue and red colors represent displacement toward and away from the satellite, respectively; while for the azimuth displacement offset map (Figure 4b), blue and red colors repre- sent displacement toward and opposite to the satellite flight direction, respectively. We interpret steep offset gradients as the surface expressions of the earthquake ruptures. In Figure 4, two sharp color discontinuities are highlighted with two solid lines, which are about 245 $\mathrm{km}$ and $60 \mathrm{~km}$, respectively. Solid lines in Figure 4 are recognized to be along Beichuan-Yingxiu fault and Guanxian-Jiangyou fault, respectively.

To further interpret the ASAR offset results, two profiles across the faults are given on the displacement offset maps of range and azimuth, respectively (lines $A B$ and $C D$ in Figure $4 \mathrm{a}, E F$ and $G H$ in Figure $4 \mathrm{~b}$ ); and their plots are shown in Figure 5. $E F$ and $A B$ straddle both Beichuan- Yingxiu fault and Guanxian-Jiangyou fault on the displacement offset maps of range and azimuth respectively, but $G H$ and $C D$ only straddle Beichuan-Yingxiu fault.

By analyzing the four profiles, we come to the following conclusions.

1) In the range field, there is a strong upheaval along the line of sight in the NW side of Beichuan-Yingxiu fault, while only a slight upheaval in the SE side. A major displacement field is localized within a zone not wider than 20 kilometers, and the amount of relative displacement near Beichuan-Yingxiu fault reaches up to about $5.9 \mathrm{~m}$. Great differences of displacement appear between two sides of BeichuanYingxiu fault, which is the major reason for generating a large-scale ruptures zone. In addition, trend of echelonment rising is a typical characteristic from the southern side of Guanxian- Jiangyou fault to the northern side of Beichuan-Yingxiu fault.

2) In the azimuth field, the movement in the NW side of Beichuan-Yingxiu fault is opposite to the satellite flight direction (red), while that in the SE side is toward the flight direction (blue). Moreover, the maximum displacement in the NW side of Beichuan-Yingxiu fault is about $1.5 \mathrm{~m}$ in the satellite flight direction, which is only a component of horizontal movement. So Beichuan-Yingxiu fault is an active fault with a stronger dextral strike-slip movement.

In sum, we have successfully located two rupture zones of Wenchuan earthquake by an offset-tracking procedure. The results show that about $245 \mathrm{~km}$ and 60 $\mathrm{km}$ in length along Beichuan-Yingxiu fault and Guanxian-Jiangyou fault were ruptured by Wenchuan earthquake respectively, and strong upheaval in range direction and horizontal movement in azimuth direction are discovered along Beichuan-Yingxiu fault. We also 
draw a conclusion that Beichuan-Yingxiu fault is an active fault with a stronger dextral strike-slip movement. The results of offset-tracking are validated by field ob-
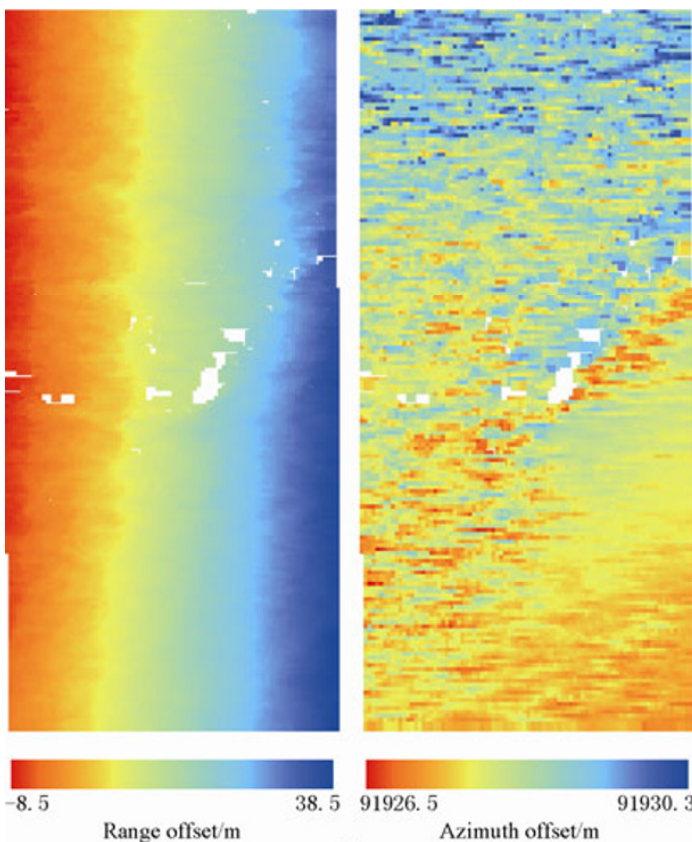

8. 5 servations of China Earthquake Administration (Xu et al, 2008). Our results complement a broader view of the rupture observations.
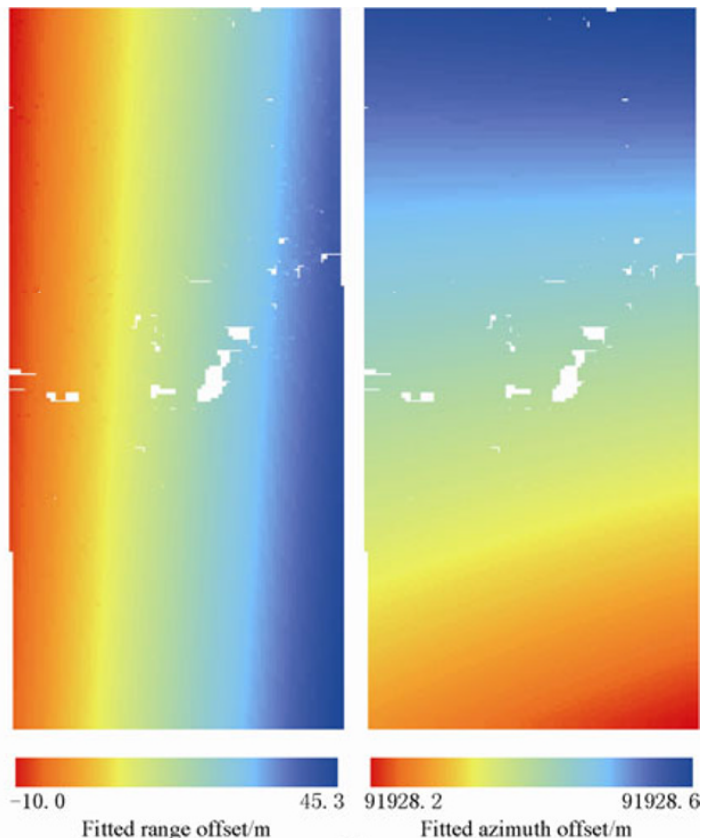

(b)

Figure 3 (a) Estimated offsets of track 290_range (left) and track 290_azimuth (right); (b) Non-coseismic components of track 290 range (left) and track 290 azimuth (right) estimated from the points far away from coseismic areas.
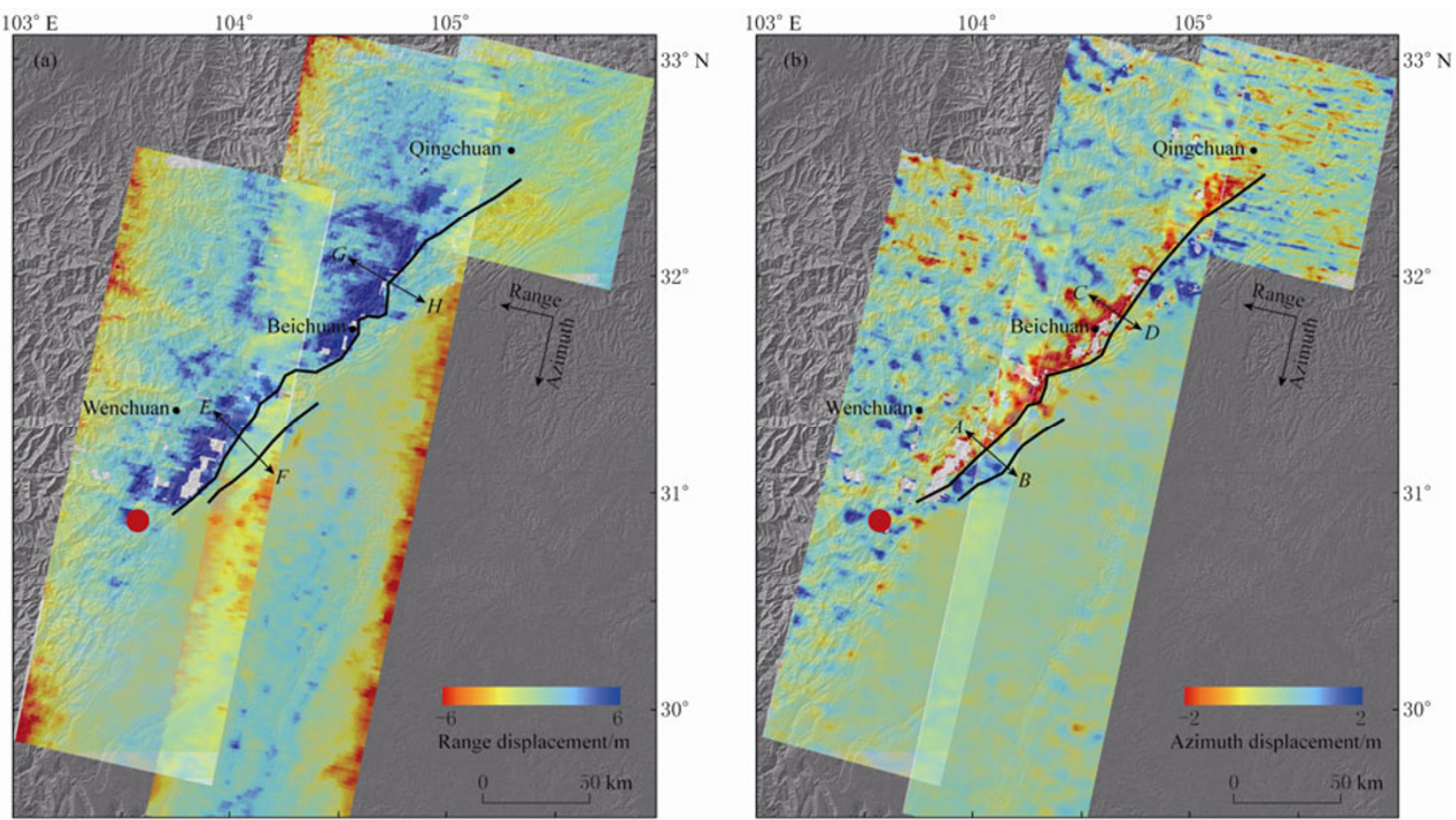

Figure 4 (a) Displacement offset map in the range direction (blue and red colors represent displacements toward and away from the satellite, respectively); (b) Displacement offset map in the azimuth direction (blue and red colors represent displacements toward and opposite to the satellite flight direction, respectively). 

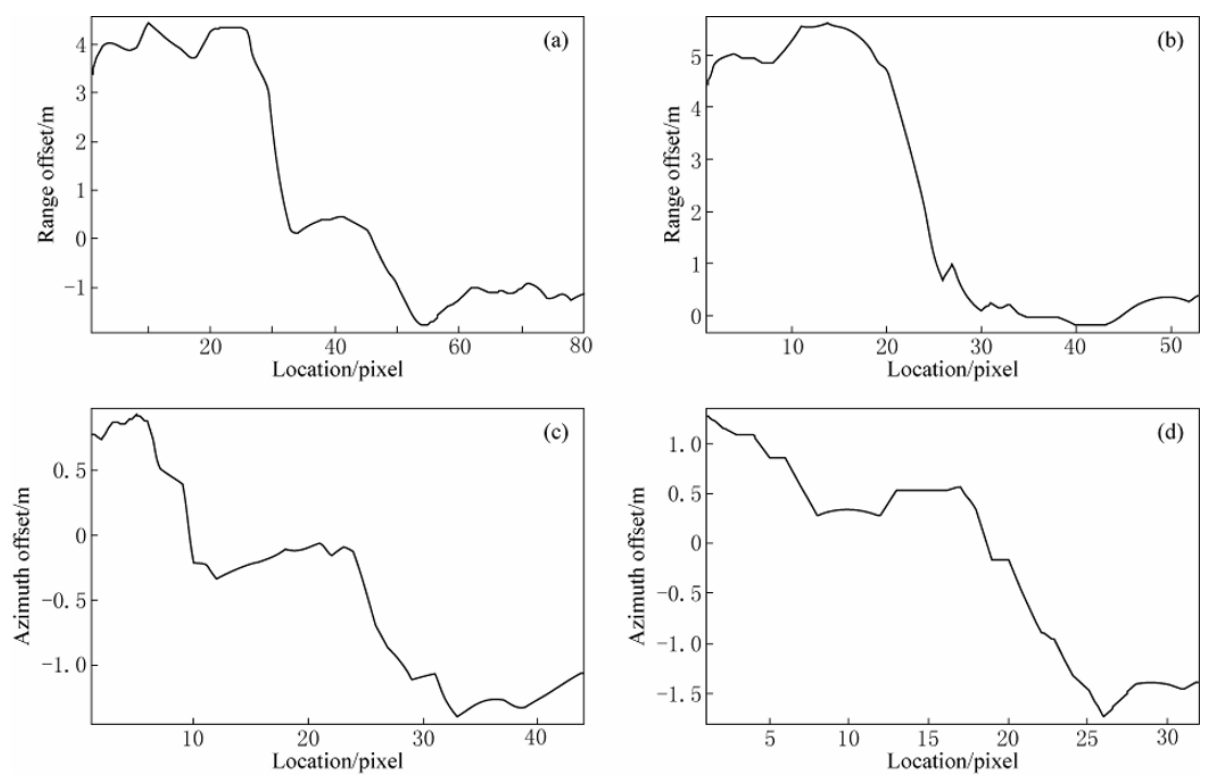

Figure 5 Plot of line $E F$ (a) and line $G H$ (b) on range displacement offset map as well as plot of line $A B(\mathrm{c})$ and line $C D(\mathrm{~d})$ on azimuth displacement offset map.

Acknowledgments Thanks are given to European Space Agency and USGS for providing ENVISAT ASAR data and ETM/TM images. We also thank anonymous reviewers and editors for their constructive comments and suggestions. This work was partially sponsored by the Special Item of Professional Fund for Basic Scientific Research of Chinese Central-Level Public-Welfare College/Institute from Chinese Finance Department (ZDJ2009-36), National Natural Science Foundation of China (40774023), National 863 Program of China (2006AA12Z150), and the project Dragon 2 (5343).

\section{References}

de Michele M, Raucoules D, Lasserre C, Pathier E, Klinger Y, van der Woerd J, de Sigoyer J and Xu X W (2010). The $M_{\mathrm{W}} 7.9$, 12 May 2008 Sichuan earthquake rupture measured by sub-pixel correlation of ALOS PALSAR amplitude images. Earth, Planets and Space (in press).

Deng Q D, Ran Y K and Yang X P (2007). Map of Active Tectonics in China (1:4 000 000). Seismological Press, Beijing, 1 (in Chinese).

Ge L L, Zhang K, Ng A, Dong Y, Chang H C and Rizos C (2008). Preliminary results of satellite radar differential interferometry for the coseismic deformation of the 12 May $2008 M_{\mathrm{S}} 8.0$ Wenchuan earthquake. Geographic Information Science 14(1): 12-19.

Kääb A (2005). Combination of SRTM3 and repeat ASTER data for deriving alpine glacier flow velocities in the Bhutan Himalaya. Remote Sensing of Environment 94(4): 463-474.
Kobayashi T, Takada Y, Furuya M and Murakami M (2009). Locations and types of ruptures involved in the 2008 Sichuan earthquake inferred from SAR image matching. Geophys Res Lett 36: L07302, doi:10.1029/ 2008GL036907.

Shan X J, Qu C Y, Song X G, Zhang G F, Liu Y H, Zhang G H and Li W D (2008). Coseismic surface deformation caused by the Wenchuan $M_{\mathrm{S}} 8.0$ earthquake from InSAR data analysis. Chinese J Geophys 52(2): 496-504 (in Chinese with English abstract).

Strozzi T, Luckman A, Murray T, Wegmüller U and Werner C L (2002). Glacier motion estimation using SAR offset-tracking procedures. IEEE Transactions Geoscience and Remote Sensing 40(11): 2 384-2 391.

Strozzi T, Wiesmann A, Sharov A, Kouraev A, Wegmüller U and Werner C (2006). Capabilities of L-band SAR data for arctic glacier motion estimation. Proceeding of 2006 International Geoscience and Remote Sensing Symposium. IEEE GRSS Press, Denver Colorado, USA, 3 816-3 819.

Sun J B, Liang F, Shen Z K and Xu X W (2008). InSAR deformation observation and preliminary analysis of the $M_{\mathrm{S}} 8$ Wenchuan earthquake. Seismology and Geology 30(3): 789-795 (in Chinese with English abstract).

Wegmüller U, Werner C, Strozzi T and Wiesmann A (2006). Ionospheric electron concentration effects on SAR and INSAR. Proceeding of 2006 International Geoscience and Remote Sensing Symposium. IEEE GRSS Press, Denver Colorado, USA, 3 731-3 734.

Werner C, Wegmüller U, Strozzi T and Wiesmann A (2005). Precision estimation of local offsets between SAR SLCs and detected SAR images. Proceeding of 2005 International Geoscience and Remote Sensing Symposium. IEEE GRSS Press, Seoul, Korea, 4 803-4 805.

Xu X W, Wen X Z, Ye J Q, Ma B Q, Chen J, Zhou R J, He H L, Tian Q J, He Y L, Wang Z C, Sun Z M, Feng X J, Yu G H, Chen L C, Chen G H, Yu S E, Ran Y K, Li X G, Li C X and An Y F (2008). The $M_{\mathrm{S}} 8.0$ Wenchuan earthquake surface ruptures and its seismogenic structure. Seismology and Geology 30(3): 597-629 (in Chinese with English abstract). 